\title{
Sensitivity Analysis of Material Deterioration on Seismic Performance Evaluation of Bridges
}

\author{
Jintao Cui ${ }^{1, a^{*}}$ and Peng Cui ${ }^{2, b}$ \\ ${ }^{1}$ School of Civil Engineering, Tianjin Chengjian University, Tianjin, China \\ ${ }^{2}$ Engineering Institute, Ocean University of China, Qingdao, Shandong province, China \\ acjt@tcu.edu.cn, b moon0917@outlook.com
}

Keywords: sensitivity analysis, seismic performance evaluation, material deterioration.

Abstract. The steel corrosion is usually displayed in the form of cracking and spalling of the concrete cover due to the expansion of corrosion products. Structural distress due to either loss of bond along the steel/concrete interface resulting from splitting of the concrete cover, or reduction of the cross-sectional area of the reinforcing bars can be caused over time. The time-variant reliability analysis and seismic fragility analysis are combined together to form a continuous fragility curve in load as well as in time. The uncertainties of material deterioration may affect the seismic performance evaluation of bridges. The tornado diagram analysis has been used here due to its simplicity and efficiency to identify sensitivity of uncertain parameters of material properties and section dimensions.

\section{Introduction}

The dynamic properties of structures such like stiffness and damping ratio are not constant but vary in time due to strength and stiffness degradation, crack, corrosion, and fatigue. This means that the probabilistic analysis does not conclude random variables only, but also functions of time, usually referred to as random or stochastic processes. As a result, the failure probability is also a function of time. In the case of seismic performance, the existing structures are apt to be deficient to earthquake events, especially if they were built in the time when the seismic design code was not fully developed. The seismic performance of an existing structure is generally evaluated considering design drawings and visual inspection data. However this approach has a limitation that the degraded material properties such as changes in stiffness and damping ratio cannot be quantitatively considered. To predict how these parameters influence the seismic performance of reinforced concrete (RC) structures, it is necessary to have the knowledge of their changes over time.

Reinforcement corrosion in concrete is the dominant cause of premature deterioration of RC structures. The structural deterioration induced by corrosion is a gradual process consisting of a few phases during the service life of RC structures, including corrosion initiation and propagation, concrete cracking, excessive deflection and final collapse due to loss of structural strength. Various theoretical frameworks have been developed to assess the service life performance of structures, including the employment of advanced reliability theories [1-4].

The numerical analysis techniques for RC deterioration model and time-variant fragility analysis are presented to form the time-variant fragility curves. The uncertainties of material deterioration may affect the seismic performance evaluation of bridges. Reduction of the number of uncertainty parameters cuts down the computational effort and cost. One way of doing this is to identify those parameters with associated ranges of uncertainty that lead to relatively insignificant variability in seismic response and then treating as deterministic parameters by fixing their values at the mean values. For ranking uncertain parameters according to their sensitivity to desired response parameters, there are various methods such as tornado diagram analysis, first order second moment (FOSM) analysis and Monte Carlo simulation. Monte Carlo simulation is computational demanding due to the requirement of a large number of simulations. The tornado diagram analysis has been used here due to its simplicity and efficiency to identify sensitivity of uncertain parameters of material properties and section dimensions. 


\section{Service Life Prediction Model}

It may be noted that, in service life prediction of RC structures affected by corrosion, the well-known Tuutti model (1982) [5] has existed for a couple of decades. The detrimental effect of corrosion on the service life of reinforced concrete structures highlights the need for the development of mathematical models of the process of corrosion and the mechanics of damage buildup. The advantage of developing a mathematical service life model is in the breadth of possible applications of such a model to a range of different materials and conditions. Even though a lot of research on reinforcement corrosion has been done in the last two decades, reliable prediction models of the expected or remaining service life of reinforced concrete structures do not exist yet. An understanding of the mechanisms and kinetics of the corrosion process as well as the rates of deterioration is the basis for making quantitative predictions of the service life of reinforced concrete structures exposed to chloride environments.

Reliable service life models can help engineers to make decisions on the type of materials used and concrete covers required to ensure the design life of the structure is met. Besides durability design, service life estimations are also useful for rehabilitation and maintenance planning of in-service reinforced concrete structures. The corrosion propagation causes the degradation of the RC structures such as loss of cross-sectional area of reinforcement, reduction of bond, crack initiation and propagation caused by expansive rust products. The deterioration process of RC structures subjected to chloride attack can be divided into three stages: (i) time to corrosion initiation, (ii) corrosion initiation to crack initiation and (iii) crack propagation causing severe cracking.

\section{Example Bridge}

The example model is a PSC-I type girder bridge as shown in Fig. 1. The example bridge is a four span bridge for which each span is $35 \mathrm{~m}$ long and the total bridge length is $140 \mathrm{~m}$. It is assumed that the example bridge is exposed to the marine environment condition for a certain long time.

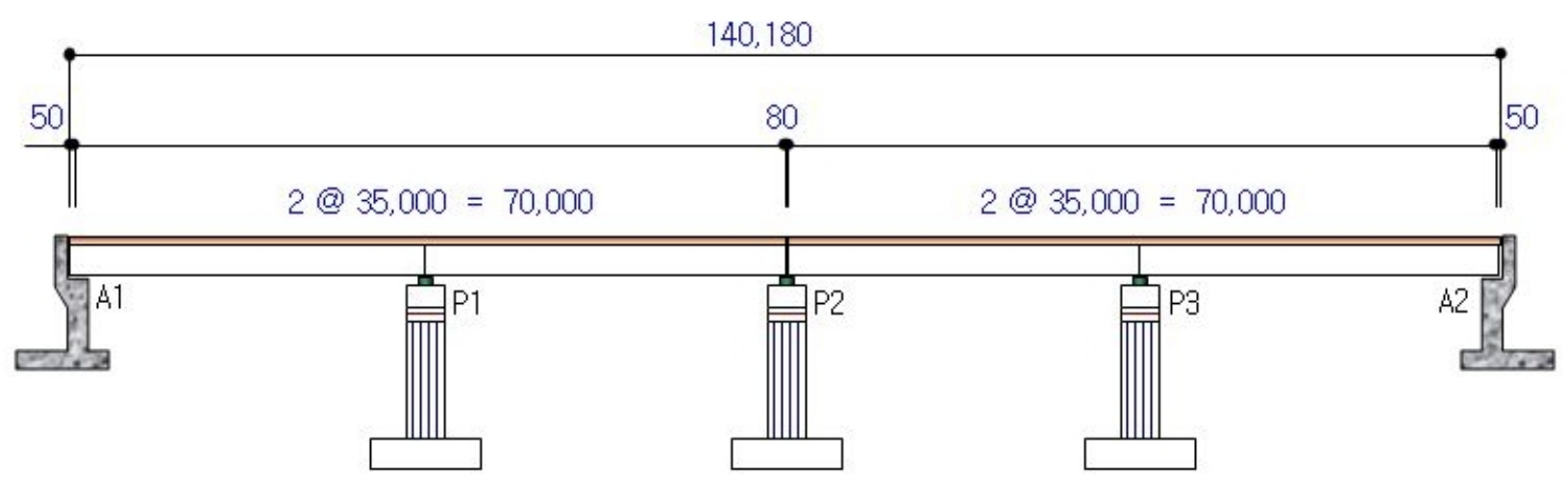

Fig. 1 Front view of the example bridge

The example bridge is modeled by the nonlinear beam-column element. The superstructure of the example bridge is modeled by elastic beam elements and the pier of the example bridge is modeled by nonlinear column elements which are divided transversally into fibers and longitudinally to segments. This type of finite element model is chosen to provide good accuracy in the calculation of the pier nonlinear behavior. For the boundary conditions, the bottom of the pier is fixed and the superstructure and substructure are connected by hinge and roller.

\section{Tornado Diagram Analysis}

For the tornado diagram analysis, all uncertain parameters are assumed as random variables, and for each of these random variables, two extreme values with 15 percentages variances corresponding to 
assumed upper and lower bounds of its probability distribution have been selected, respectively. It can be observed that these extreme values come from the normal distribution assumption, mean plus standard deviation and mean minus standard deviation representing their upper and lower bounds, respectively. Using these two extreme values for a certain selected random variables, the seismic response of the example model bridge subjected to a specific earthquake ground motion has been evaluated for both cases, while all other random variables have been assumed to be deterministic parameters with their mean values. The relative difference of these two response values in percentage corresponding to the two extreme values of that random variables is calculated, which is termed as swing of the response corresponding to the selected random variable. This calculation procedure has been repeated for all random variables of material properties and section dimensions in question. Finally, these swings have been plotted in a figure from the top to the bottom in descending order according to their size to demonstrate the relative contribution of each variable to the response under question. It is noteworthy that longer swing implies the corresponding variable has larger effect on the response than those with shorter swing.

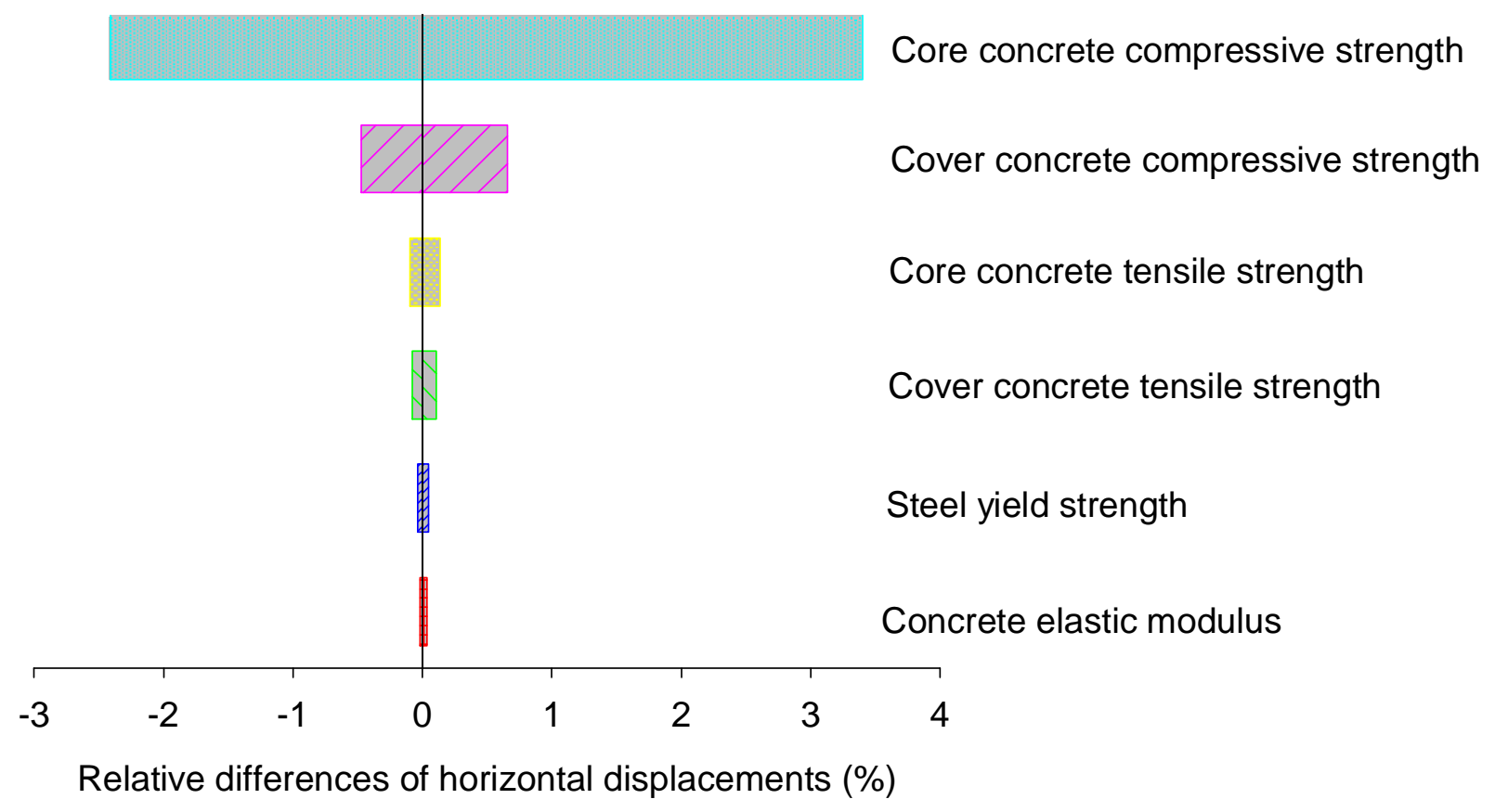

Fig. 2 Tornado diagram considering uncertainty of material properties

For tornado diagram analysis, the concrete (core and cover concrete separately) and steel strength parameters are selected to compare their sensitivities to seismic displacement response of the example bridge and the artificial earthquake which has a PGA at 0.6g is used. As shown in Fig. 2 for the tornado diagram, the horizontal line indicates the relative difference of maximum transverse displacement in percentage, which is calculated by comparison with the value of $11.794 \mathrm{~mm}$ corresponding to the maximum transverse displacement that calculated using the mean values of all random variables. The length of swing (horizontal bar) represents the variation in the relative difference of maximum transverse displacement in percentage due to the variation in the respective random variables.

For the parameters of concrete strength, cover depth, reinforcing size, horizontal spacing and corrosion rate, corrosion rate is the most significant parameter in determining the time to cracking of the cover concrete. The corrosion rate is the key to predicting the time to cracking, which is largely controlled by the rate of oxygen diffusion to the cathode, resistivity of the pore solution, and temperature. It is well recognized that both the corrosion rate and cover depth have a large effect on the time to cracking. Fig. 3 shows the reduction of a steel rebar area according to different corrosion rates. 


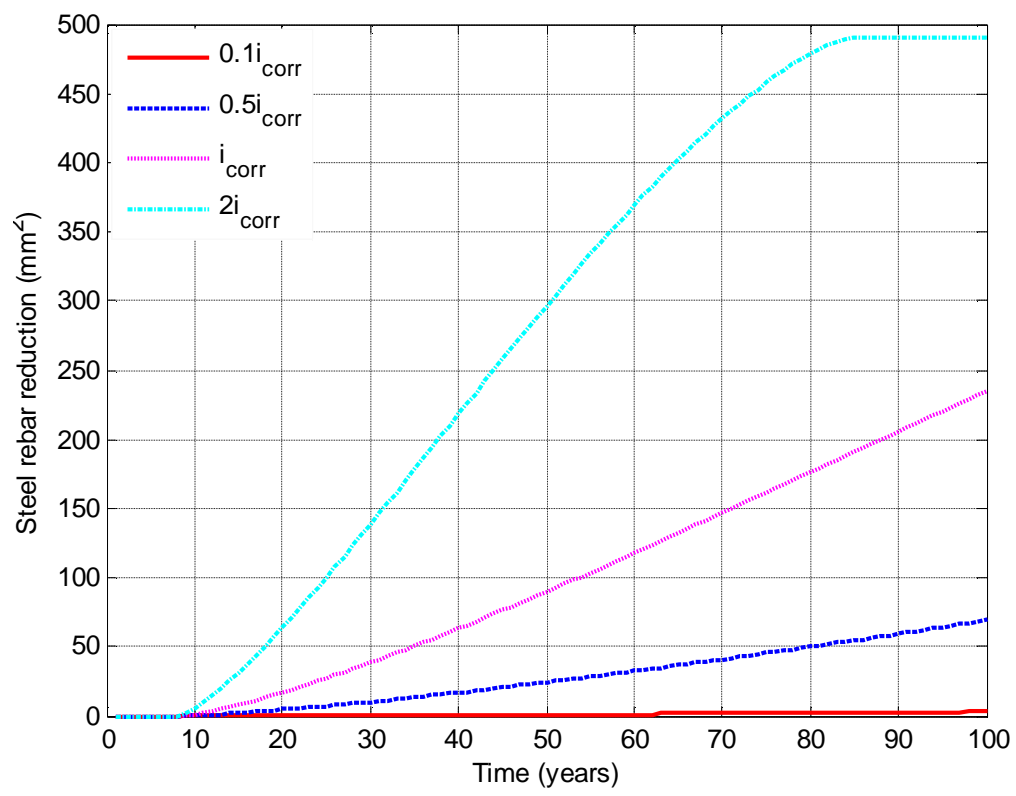

Fig. 3 Sensitivity analysis of steel rebar reduction considering uncertainty of corrosion rate

\section{Conclusions}

It can be noted that the core and cover concrete compressive strength are the two largest contributors of the response variability. Other parameters such as the core and cover concrete tensile strength, steel yield strength and concrete elastic modulus have less contributors of the response variability. It can be also noticed from Fig. 2 that nearly all swings are asymmetric about the vertical lines, which implies the high nonlinearity of the problem. In other words, the same amount of a positive and a negative change in these parameters does not produce the same amount variation in maximum transverse displacement.

\section{Acknowledgements}

This work was financially supported by General Projects in Tianjin Research Program of Application Foundation and Advanced Technology (13JCYBJC39100). The authors would like to appreciate for the financial supports.

\section{References}

[1] Maage, M., Helland, S., Poulsen, E., Vennesland, O., \& Carlsen, J. E. (1996). Service life prediction of existing concrete structures exposed to marine environment. ACI Materials Journal, 93(6), 602-608.

[2] Frangopol, D. M., Lin, K. Y., \& Estes, A. (1997). Reliability of reinforced concrete girders under corrosion attack. Journal of Structural Engineering, 123(3), 286-297.

[3] Engelund, S., Sorensen, J. D., \& Sorensen, B. (1999). Evaluation of repair and maintenance strategies for concrete coastal bridges on a probabilistic basis. ACI Materials Journal, 96(2), 160-166.

[4] Li, C. Q. (2004). Reliability Based Service Life Prediction of Corrosion Affected Concrete Structures. Journal of Structural Engineering, 130(10), 1570-1577.

[5] Tuutti, K. (1982). Corrosion of steel in concrete. Stockholm: Swedish Cement and Concrete Research Institute. 